\title{
Eficacia del método Ponseti en el tratamiento del pie equinovaro
}

\author{
Beatriz Naranjo Morales ${ }^{1}$
}

Fecha de recepción: 4 de abril de 2018 / Fecha de aceptación: 11 de diciembre de 2018

Resumen. Contexto: El pie equinovaro es una deformidad musculoesquelética congénita caracterizada por la presencia de cavo, varo, adducto y equino. Afecta a 1-7 de cada 1000 nacimientos. Un pie zambo no tratado puede generar dolor y discapacidad durante la vida de la persona. El método Ponseti se ha convertido en la principal modalidad de tratamiento para el manejo del pie zambo produciendo buenos resultados a largo plazo. El objetivo de este manuscrito fue evaluar la eficacia del método Ponseti y la evolución de los pacientes en función de las tasas de recurrencia, tenotomías y cirugías asociadas. También se determinó si la edad de los niños influía en el éxito del tratamiento.

Métodos: Se realizó una estrategia de búsqueda a través de Pubmed hasta abril de 2018. Los términos de búsqueda incluyeron pie zambo, método Ponseti y pie equinovaro. Se incluyeron metaanálisis, revisiones sistemáticas, ensayos clínicos y series de casos. También se examinaron las referencias bibliográficas de los artículos seleccionados.

Resultados: Veintisiete artículos fueron incluidos en esta revisión. La literatura actual muestra una tasa de éxito del $78-92 \%$ utilizando el método Ponseti.

Conclusión: El método Ponseti ofrece resultados alentadores en términos funcionales y estéticos, así como disminución del grado de corrección quirúrgica cuando los resultados no sean los esperados. Se necesita más investigación para generar evidencia de mayor calidad con mayores tamaños muestrales y unificación a la hora de medir la severidad de la deformidad. Otra limitación encontrada fue la ausencia de la definición de fracaso de tratamiento y sus correspondientes motivos.

\section{[en] Ponseti method efficacy in treatment of clubfoot}

Abstract. Background: Clubfoot is a congenital musculoskeletal deformity characterized by heel varus, hindfoot equinus, mid-foot cavus and forefoot adduction. It is the most common defect that affects between 1 and 7 births in every 1000. Left untreated, clubfoot may lead to pain and disability throughout the person's life. Ponseti method has become the main treatment modality for the management of clubfoot producing good long-term results. The aim of this manuscript was to determine the efficacy of the Ponseti method for the treatment of CTEV and to evaluate the evolution of patients based on the recurrence rate, tenotomies rates and surgeries. Also determine if the age of children influences the success of the treatment.

Methods: A search strategy completed examined Pubmed from inception to April 2018. Search terms included clubfoot, Ponseti method and equinovarus. Meta-analysis, systematic reviews, randomized control trials and case series were included. The reference lists of the selected articles were also examined.

Results: Twenty-seven articles were included in this review. The current literature shows a success of the Ponseti method of $78-92 \%$.

Conclusion: Ponseti treatment for clubfoot has encouraging results in terms of attaining a functionally and cosmetically foot and lessening the extent of surgical correction in cases in which it does not reach the expected results. Further research is needed to generate higher quality evidence with larger sample sizes which evaluate the severity of the deformity with the same measures. Another lack was the absence of a definition of treatment failure with the reasons.

Sumario: 1. Introducción 2. Materiales y métodos 3. Resultados 4. Discusión 5. Conclusión 6. Bibliografía.

\footnotetext{
Grado en Podología. Universidad Complutense de Madrid. Calle Goya 56, Telde. Las Palmas. 35214.

beatriznaranjomorales@gmail.com
} 
Cómo citar: Naranjo Morales, B (2018) Eficacia del método Ponseti en el tratamiento del pie equinovaro, en Revista internacional de ciencias podólogicas 13(1), 99-113.

La autora declara no tener ningún tipo de interés económico o comercial.

\section{Introducción}

La primera referencia al tratamiento del pie equinovaro congénito fue descrita por Hipócrates $(400 \mathrm{aC})$ el cual mencionó manipulaciones repetidas y suaves seguidas de inmovilizaciones. En 1836, Guerin fue el primer médico en usar yeso, seguido por Kite quien abogó en 1932, manipulaciones repetidas seguidas de inmovilizaciones con yeso, esta combinación se conoce como método Kite (Kite JH, 1939) (1).

No fue hasta 1940, cuando, Ponseti después de estudios profundos, desarrolló y perfeccionó su régimen de tratamiento en la Universidad de Iowa (1,2). Ignacio Ponseti (1914-2009) fue un cirujano ortopédico de origen español afincado en Estados Unidos. Él describió por primera vez las férulas de abducción, la resección del tendón de Aquiles y la transferencia del tendón tibial anterior para posibles recidivas (Ponseti IV, 1963; Ponseti IV, 1972; Ponseti IV \& Smoley EN, 2009). En 1972 publicó una descripción más detallada $\mathrm{y}$, posteriormente, publicó un manuscrito de los errores más comunes que observaba cuando su método era aplicado por otras personas (Ponseti IV, 1997)(3,4).

El pie equinovaro congénito (PEVC) o también denominado "pie zambo" es una de las deformidades congénitas del pie más comunes que ocurre en aproximadamente 1 de cada 1000 nacimientos cada año (WynneDavies, 1965)(5)2.

La OMS define anomalías congénitas como aquellas anomalías estructurales o funcionales que suceden durante la vida intrauterina y se detectan durante el embarazo, en el parto o en un momento posterior de la vida. Se denominan también defectos de nacimiento, trastornos congénitos o malformaciones congénitas.

El pie equinovaro consiste en una compleja deformidad tridimensional caracterizada por la presencia de un pie cavo, varo, adducto y equino (CAVE), cuya severidad varía de un paciente a otro $(6,7)$. Esta deformidad hace que los pacientes apoyen por el lado externo del pie, generando hiperqueratosis, posibles infecciones en la piel y huesos, artritis y limitaciones significativas en la movilidad. (Dobbs MB, 2009; Radler C, 2013; Ponseti IV, 1996)(8,9).

Más de 2.2 millones de niños al año presentan esta deformidad rígida del pie al nacer (Shabtai, Specht \& Herzenberg, 2014) (7). Alrededor del $80 \%$ de los casos se producen en países en vías de desarrollo, donde los recursos para afrontar dicha afección son escasos (Morcuende AJ, 2006; Saltzman MH, 2009) (10).

La incidencia más alta del pie zambo se localiza en Nueva Zelanda con una prevalencia de 6-7 por cada 1000 recién nacidos; una prevalencia similar a la reportada en la población polinesia. Por el contrario, la prevalencia más baja se halla en China: 0,57 por 1000 nacimientos (Werler MM, 2013; Cartlidge IJ, 1983; Beals RK, 1978) (11).

La prevalencia en varones es mayor a la de las mujeres, con una proporción 2:1; asimismo, es bilateral en el $50 \%$ de los casos (Beals RK, 1978; Dobbs MB, 2012, Foster A, 2007; Dobbs MB, 2009). Además, se afirma que el pie derecho se ve más afectado que el izquierdo (Wynne-Davies R, 1964) (8).

Acorde con un estudio realizado en Sudáfrica por Grimes CE, Holmer $\mathrm{H}$ y colaboradores, se estimó el gasto total en la realización del método Ponseti y dictaminaron que se trata de un método rentable, que debería aplicarse en el resto de países dado que supone una deformidad con significantes consecuencias sociales y económicas (12).

En la última década, el método Ponseti se ha extendido ampliamente por todo el mundo y el número de publicaciones acerca de esta temática ha aumentado exponencialmente. 


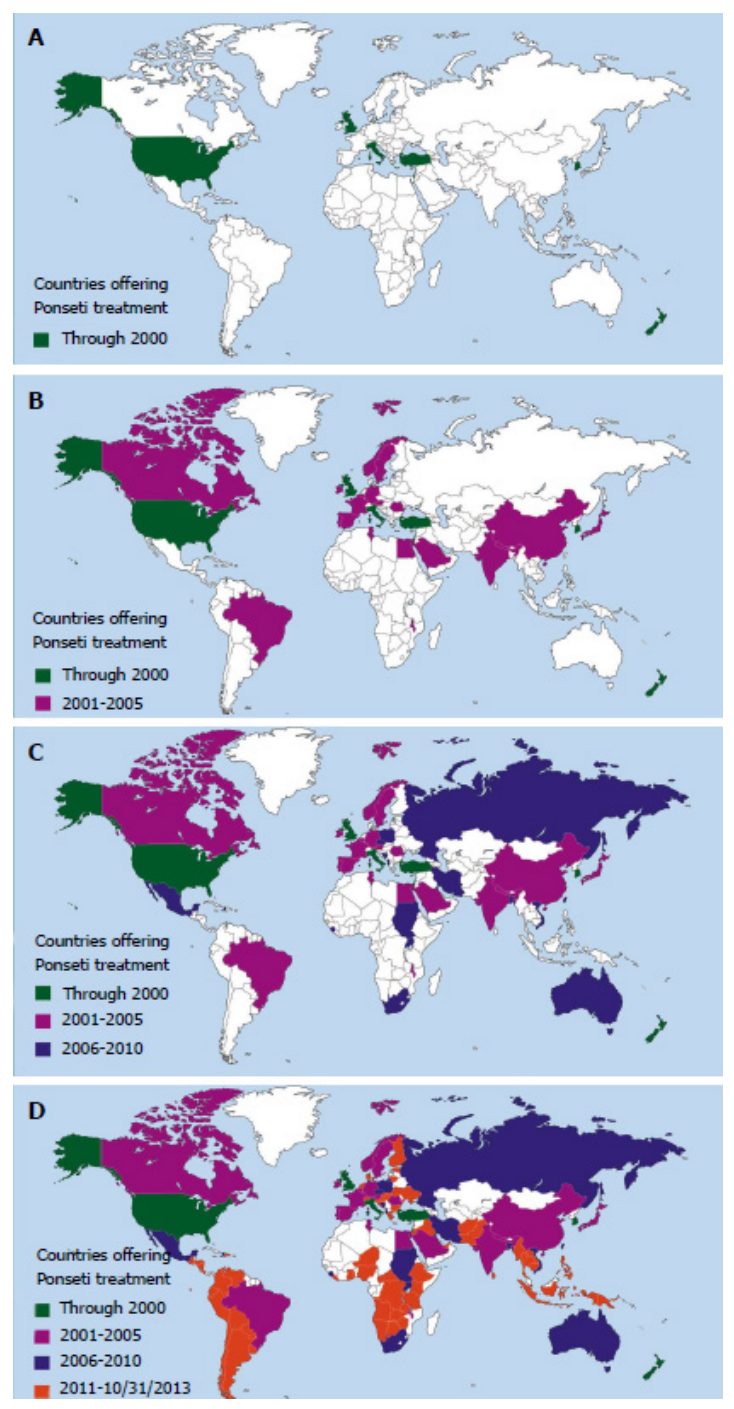

Figura 1. Orden de aparición del método Ponseti 2000-2013. Los países representados en blanco son los que son objeto de mayor formación y educación del método. (2)

Es importante conocer el desarrollo de los miembros inferiores en el feto, éstos aparecen en la $4^{\mathrm{a}}$ semana del periodo gestacional. El pie aparece en la $4^{\mathrm{a}}-5^{\mathrm{a}}$ semana y en la $6^{\mathrm{a}}$, estará en equino y en posición invertida. Los dedos quedarán formados a partir de la $9^{\text {a }}$ semana y la $1^{\text {a }}$ y $5^{\mathrm{a}}$ cabeza metatarsal estarán plantarflexionados; además, en este periodo, se forma el arco transversal del pie. Al mismo tiempo, el astrágalo y el calcáneo están conformando la articulación subastragalina. Durante doce semanas el pie está en posición de supinación. En la semana 28 el pie alcanzará una posición neutra (Bernhardt DB, 2007) (8).
Biomecánicamente, las deformidades del pie zambo ocurren debido a anormalidades de los huesos del tarso, ligamentos, articulaciones $y$, retracción y atrofia de la musculatura de la pantorrilla viéndose disminuido su volumen. El astrágalo se encuentra en flexión plantar, y la cabeza y el cuello del astrágalo se desplazan hacia medial. En algunos pies equinovaros, el cuello del astrágalo puede estar ausente o acortado. El calcáneo estará en adducción y en inversión medialmente girado por debajo del astrágalo. En casos severos de pie zambo, el escafoides se desplazará hacia medial y se articulará con la cara medial de la cabeza del astrágalo (8).

Se desconoce con exactitud la etiopatogenia del pie equinovaro, sin embargo, se piensa que se trata de una causa multifactorial. Se proponen dos teorías en la aparición de la malformación: la primera, un defecto del plasma del astrágalo que causa plantarflexión e inversión del mismo, y la segunda, un defecto de tejidos blandos que consecuentemente causa cambios en el hueso (Kelly, 2013) (11).

Las causas pueden ser vasculares, ambientales, genéticas, mecánicas (posición anormal en el útero) o anatómicas. Un componente genético es sugerido por la concordancia del $33 \%$ en gemelos y la recurrencia familiar en el $25 \%$ de 10 s casos (Lochmiller C, 1998) (9). Alrededor del $20 \%$ de las deformidades del pie zambo están asociadas a otras anomalías congénitas tales como: artrogriposis, mielodisplasia, mielomeningocele, distrofia miotónica, síndrome de Edwards (trisomía del cromosoma 18), síndrome de Down, síndrome de Larsen y síndrome de FreemanSheldon (Brewer C, 1998; Foster A, 2007; Siapkara A, 2007) (9).

Existen varios factores de riesgo asociados al pie zambo entre ellos el sexo masculino, madre o padre fumadores durante el embarazo, diabetes gestacional, estado civil de la madre, educación materna, edad materna y paridad $(7,8,10)$.

Según Melissa Palma y colaboradores, el nacimiento de los recién nacidos en los meses de invierno y una temprana edad paterna se correlacionó con mayor riesgo de desarrollar un pie zambo (10).

Asimismo, se vio que el contenido apropiado de ácido retinoico en el embrión de los vertebrados es esencial para un correcto desarrollo. El ácido retinoico es un 
metabolito oxidativo de la vitamina A que controla múltiples procesos biológicos, entre ellos el desarrollo embrionario, de ahí que su deficiencia o su exceso puedan causar malformaciones congénitas tales como dismorfogénesis de las extremidades. De ahí surge la hipótesis de que un inadecuado contenido de ácido retinoico puede desarrollar un pie zambo, al modular de manera global el crecimiento fetal. DelgadoBaeza y colaboradores realizaron un estudio experimental con ratas, para estudiar esta asociación a través de un método de doble tinción para la osificación esquelética, y finalmente constataron, que tanto el astrágalo como el calcáneo estaban peor osificados y resaltaron que la severidad patológica de la malformación del pie zambo deriva generalmente de estos dos huesos (DelgadoBaeza, 1999). Z-Y Liu y cols. comprobaron la pobre osificación de los metatarsianos, la ausencia de falanges distales y la ausencia de los centros de osificación en fémur, tibia y peroné en un modelo animal experimental tras la administración de ácido retinoico (13).
El pie zambo se puede clasificar en cuatro tipos según las causas y la respuesta al tratamiento:

1. Postural

2. Idiopático: "verdadero pie zambo"

3. Neurogénico

4. Asociado a síndromes: de tipo rígido (Carroll NC, 2012) (8).

Un sistema de clasificación ideal debe ser reproducible, lo suficientemente práctico para su uso en un entorno clínico y debe predecir el tratamiento apropiado en una etapa temprana. Aunque los sistemas de clasificación de Pirani y la de Dimeglio son los más utilizados, existen diferentes herramientas para la evaluación clínica de los pacientes con pie equinovaro (Dimeglio A, 1995; Gao R, 2014; Shaheen; 2012).

- Escala de clasificación de Pirani: documenta la gravedad de la deformidad. Valora 6 signos clínicos, siendo 0 (normal), 0,5 (moderado) y 1 (severo), siendo la puntuación de 6 la deformidad más grave.

Tabla 1. Clasificación de Pirani $(14,24)$

\begin{tabular}{|l|l|}
\hline \multicolumn{1}{|c|}{ Variable } & \multicolumn{1}{c|}{ Puntuación } \\
\hline $\begin{array}{l}\text { Retropié: } \\
\text { Pliegue posterior }\end{array}$ & $0-3$ \\
- Equino rígido & $\bullet 0,0^{\prime} 5,1$ \\
\hline$\quad$ Talón vacío & $\bullet 0,0^{\prime} 5,1$ \\
\hline $\begin{array}{l}\text { Mediopié: } \\
\text { Pliegue medial }\end{array}$ & $0,0^{\prime} 5,1$ \\
\hline Curvatura del borde lateral & $\bullet 0,0^{\prime} 5,1$ \\
\hline Cobertura astrágalo & $\bullet 0,0^{\prime} 5,1$ \\
\hline Puntuación total: & $-0,0^{\prime} 5,1$ \\
\hline
\end{tabular}

- Clasificación de Dimeglio: se basa en la severidad de la deformidad. El puntuaje de Dimeglio tiene un máximo de 20 puntos, clasificando la deformidad como benigna, moderada, severa o muy grave.

Tabla 2. Clasificación de Dimeglio $(14,24)$

\begin{tabular}{|c|l|l|l|}
\hline Grado & \multicolumn{1}{|c|}{ Tipo } & \multicolumn{1}{c|}{ Puntuación } & \multicolumn{1}{c|}{ Reductibilidad } \\
\hline I & Benigno & $<5$ & $>90^{\circ}$. Reductible. \\
\hline II & Moderado & $5-10$ & $>50^{\circ}$. Reductible, parcialmente rígido. \\
\hline III & Severo & $10-15$ & $<50^{\circ}$. Parcialmente reductible. \\
\hline IV & Muy severo & $15-20$ & $<10^{\circ}$. Pie rígido. \\
\hline
\end{tabular}




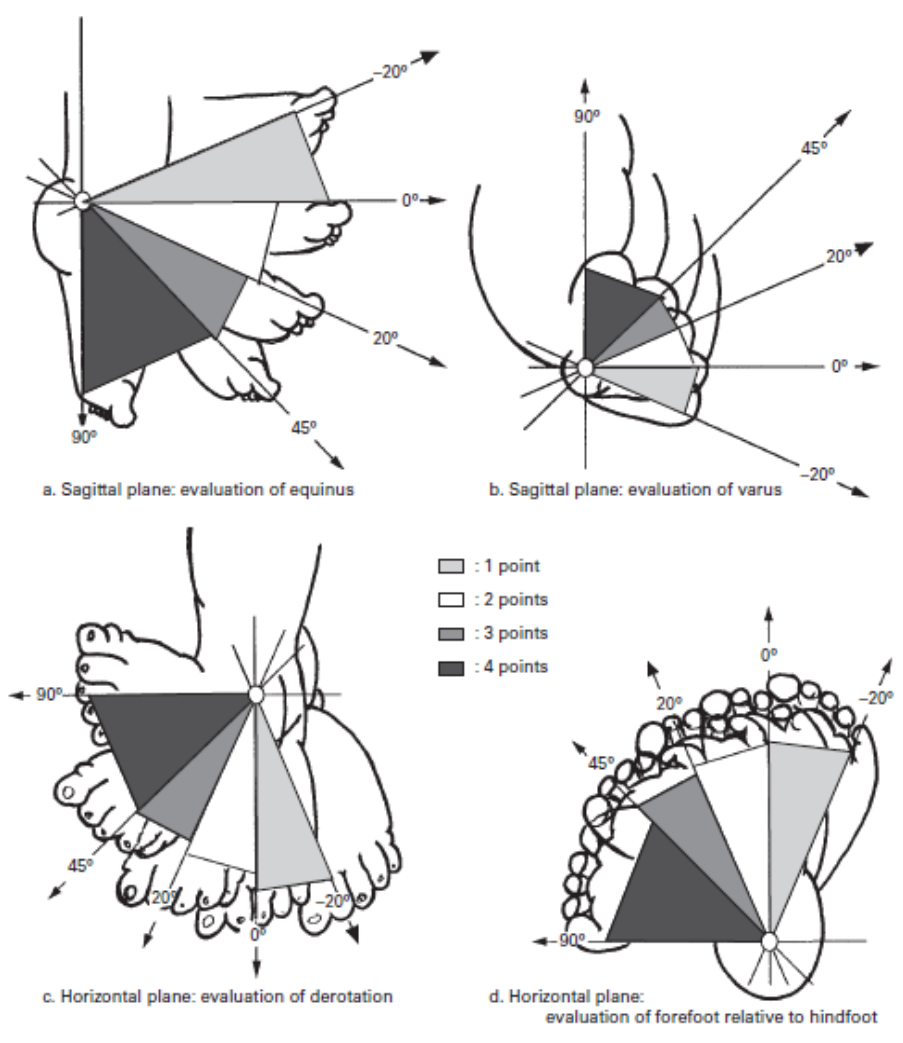

Figura 6. Diagrama de la evolución del pie equinovaro congénito utilizado por Dimeglio y colaboradores.

- Escala de Harrold Walker: considera la capacidad de corregir la deformidad. Se basa en un examen del pie a través de una estimación angular, de forma que, si el pie se puede mantener en una posición neutra hablaríamos de grado I, si hay presencia de equino o varo rígido de $<20^{\circ}$ estaríamos ante un grado II y $>20^{\circ}$ sería grado III.

- Escala de Catterall: describió cuatro patrones en base a nueve medidas según la evolución de la deformidad, ésta se clasificó como de resolución, causada por una contractura del tendón o de la articulación o secundaria a una falsa corrección (14-16).

Tanto la clasificación de Pirani como la de Dimeglio representan una evaluación clínica basada en la subjetividad que podría estar acompañada de errores de los examinadores o de una variabilidad interobservador ${ }^{(17)}$.

En la última década, la gestión conservadora ha ganado importancia en la corrección de la deformidad del pie zambo, de hecho, una revisión sistemática realizada en 2011, mostró que existen pruebas contundentes de que el método Ponseti es el régimen de tratamiento más exitoso para el pie zambo congénito, con una tasa de corrección inicial de alrededor del $90 \%$ en la mayoría de los estudios (Jowett CR, 2011) (3).

El método Ponseti está basado en manipulaciones pasivas y enyesados continuados, y supone el tratamiento más exitoso hasta la fecha, para el tratamiento del pie equinovaro y para la prevención $\mathrm{y}$ tratamiento de recidivas (3).

El método Ponseti consta de dos fases:

1. Fase de corrección: Inicialmente se recomiendan manipulaciones breves y suaves para estirar las estructuras y, además, para tener una idea de la flexibilidad del pie y la cantidad de corrección que se puede lograr con el yeso. Las manipulaciones se harán alrededor del astrágalo y posteriormente, se mantendrán en esta posición con un yeso parís que llega hasta la rodilla, el cual se cambia semanalmente. En el primer enyesado se supinará el antepié de forma que se alinee con retropié y se disminuya 
el cavo, el pie nunca deberá pronarse. En los siguientes enyesados, se realizará una abducción pura con contrapresión a nivel del cuello de astrágalo, el astrágalo se estabiliza y la mortaja tibioastragalina no podrá girar. El calcáneo no debe tocarse para no bloquear su movimiento, debe oscilar libremente y por tanto, abducir, evertir y dorsiflexionar. En caso de que el calcáneo quede bloqueado, las fuerzas actuarán en las articulaciones de Chopart y de Lisfranc, de forma que la abducción ocurre a nivel de mediopié pudiéndose desarrollar un pliegue lateral. Según el protocolo, los yesos se cambiarán una vez por semana; sin embargo, Morcuende et al. describieron resultados similares con cambios de enyesado cada 5 días (Morcuende JA, 2005). El deslizamiento del pie dentro del yeso ha sido considerado como un importante factor en el desarrollo de un pie zambo complejo (Ponseti IV, 2006) (3).

La Tenotomía percutánea del tendón de Aquiles (TpA) es uno de los componentes principales del método Ponseti y normalmente es necesaria para corregir el equino residual y minimizar el riesgo de recidiva (Ponseti IV, 2006) (3). Ponseti recomendó realizar la TpA bajo anestesia local, tras previa palpación y ejecutarla cuando el pie lograse alcanzar $60^{\circ}$ de abducción y $15-20^{\circ}$ de dorsiflexión (Laaveg, 1980). En caso de realizarse antes de $60^{\circ}$ de abducción y sin una correcta alineación de la subastragalina, el retropié probablemente permanecerá sin corregir (Ponseti IV, 1997). No obstante, en ocasiones es difícil valorar el ángulo de flexión dorsal de tobillo en un pie pequeño, y algunos autores, toman como referencia radiográfica la presencia de un ángulo talocalcáneo lateral superior a $80^{\circ}$ como subsidiario de la realización de una TpA. Tener especial cuidado a la hora de realizar el corte, de forma que no se haga demasiado próximo al nervio tibial, a los vasos tibiales y peroneos, así como evitar lesiones de la cúpula astragalina. Existen varios trabajos que anuncian las complicaciones derivadas de la realización de la tenotomía, entre las que destacan: hemor- ragia grave por lesión de los vasos peroneos, pseudoaneurisma y daño del paquete neurovascular con necesidad de posterior ligadura y reparación primaria del nervio (Dobbs MB, 2004; Burghardt RD, 2008; Changulani M, 2006) (3). Tras la tenotomía se aplica un yeso largo de pierna colocado en $70^{\circ}$ de abducción y $5-10^{\circ}$ de dorsiflexión (Dobbs y Gurnett, 2009) (24).

2. Fase de mantenimiento: implica el uso de una ortesis de pie-tobillo (AFO) o bien, de un zapato unido a una barra de Denis Browne, el zapado debe ser alto, abierto, ajustado y de horma recta (Dobbs MB, 2004; Thacker MM, 2005; Abdelgawad AA, 2007) (21). El pie afectado se mantiene en $60-70^{\circ}$ de rotación externa y en $10-15^{\circ}$ de flexión dorsal durante 23 horas diarias durante 3 meses, seguido de su uso nocturno hasta los 3-4 años de edad (Staheli L, 2009; Ponseti IV \& Smoley EN, 2009; Desai L \& Oprescu F, 2010; Janicki JA, 2011(15,23).

Los tejidos blandos del tobillo como el músculo tibial anterior, músculo tibial posterior, músculo flexor largo de los dedos, músculo flexor largo del hallux, ligamento lateral interno y ligamento de Spring, están bajo estiramiento estático gracias a la AFO. Estos hallazgos sugieren que la AFO no sólo puede mantener la corrección lograda por el yeso sino que también reduce la rigidez de los tejidos blandos así como aumenta el rango de movimiento del tobillo (Staheli L, 2009) (20).

Los datos iniciales del grupo de la Universidad Iowa mostraron una tasa de recaída del 56\%; sin embargo, cuando el protocolo de uso de las férulas de abducción pasó de ser de 4 años en lugar de 2 años, la tasa de recurrencia mostró una reducción drástica (21).

Después de un correcto manejo, el paciente conseguirá un pie plantígrado funcional, indoloro sin hiperqueratosis y sin necesidad de zapatos correctivos (21). En comparación con otras técnicas, se ha visto que la mayor ventaja que presenta el método Ponseti es el grado de movilidad alcanzado al final del tratamiento (Sizínio HK, 2009) (1)

Hoy en día, el método Ponseti se considera el gold standard para la deformidad del pie 
zambo entre cirujanos ortopédicos (Ippolito \& Farsetti, 2003; Herzenberg \& Radler, 2002; Morcuende; 2006) (5). No obstante, los seguimientos clínicos mostraron que no todas las deformidades de pie zambo responden de manera similar a este método, hay cierto grado de resistencia al tratamiento y muchas deformidades aparentemente curadas, tienden a reaparecer (17). La repetición del método Ponseti es la base del tratamiento para la recidiva de pie zambo (Liu Y, 2016) (22).

El método francés o del fisioterapeuta se centra principalmente en el estiramiento y la estimulación de los músculos del lado medial del pie mediante manipulaciones correctivas pasivas diarias. Para mantener la reducción lograda, se colocarán férulas $\mathrm{y} / \mathrm{o}$ vendajes elásticos o inelásticos durante 1-3 meses. El propósito del método francés es el mismo que el del método Ponseti, es decir, conseguir mayor flexibilidad del pie, reducir el dolor y minimizar el número de recidivas y cirugías. El tratamiento quirúrgico estaría indicado ante fracaso del tratamiento ortopédico o cuando no se observen signos de mejoría (Dimeglio A, 2004; Bensahel H, 1994; Seringe R, 1990) (8).

El método Copenhagen fue desarrollado en el Hospital ortopédico de dicha ciudad y consiste en la manipulación mediante la flexión y enyesado diario. Los músculos evertores del pie como los peroneos deberán ser estimulados, este método recomienda realizar los ejercicios de estiramiento 5 veces al día. Una vez corregido, se mantendrá con un vendaje y será valorado periódicamente hasta la madurez esquelética (Steinman S, 2009; Ward S, 2015) $(6,8)$.

Las radiografías del pie son importantes para verificar regularmente el impacto del tratamiento ortopédico en la anatomía del pie, no es posible controlar el pie clínicamente sin tener en cuenta la anatomía radiológica subyacente. Cualquier falta de divergencia entre el astrágalo y el calcáneo es un signo de corrección incompleta, por lo que, las radiografías se harán durante el tratamiento y el seguimiento temprano (6).

Sin embargo, según Ponseti, las radiografías simples no evalúan el pronóstico y la gravedad del pie zambo, por lo que serán la ecografía, la tomografía computerizada (TAC) y la resonancia, las pruebas complementarias de elección para la evaluación del pie equinovaro (Roche, Mattingly \& Talkawar, 2006; Farsetti, De Maio \& Russolillo, 2009) (5). Considerando el coste de estas pruebas diagnósticas y que algunas de ellas deben realizarse bajo anestesia general en recién nacidos, para la obtención de imágenes de calidad satisfactoria, la ecografía parece ser la prueba diagnóstica de elección por su amplia disponibilidad, su bajo coste y su reproductibilidad (Aurell Y, 2005; Hamel J, 1996) (17).

A este respecto, buscando un parámetro adecuado que pueda ser fácilmente examinado por un clínico, existe la presunción de que la displasia de los huesos tarsales va acompañada de deformidad de pie zambo, siendo el astrágalo el más afectado (Chandrakanth U, 2016) (17).

A nivel mundial, el tratamiento conservador se considera el tratamiento de primera elección para la corrección del pie zambo, el cual es, claramente inevitable y debe comenzar lo antes posible tras el nacimiento del bebé, para conseguir el mejor resultado posible (Ponseti \& Campos, 2009; Cooper \& Dietz, 1995; Faulks \& Richards, 2009; Bensahel, 1994; Jowett, 2011) $(4,6)$. El tratamiento en infantes resulta desafiante debido a la rigidez que presenta el pie y a la existencia de alteraciones en el patrón de la marcha (19). Existe un consenso que dictamina que el tratamiento debe comenzar en el primer mes de vida; excepto en casos de bebés prematuros, donde se recomendó no iniciar el tratamiento en la Unidad de Cuidados Intensivos Neonatales (UCIN) y comenzarlo a posteriori, cuando el pie haya crecido y el neonato se haya estabilizado (Böhm S, 2013) (3).

Los objetivos del tratamiento incluyen funciones de marcha normal, ausencia de dolor y un apoyo plantígrado del pie (Dobbs, Morcuende \& Gurnett, 2000; Laaveg \& Ponseti, 1980; Dimeglio, 2012; Bensahel, 1994; Joweet, 2011) (5).

Un bajo nivel educativo de los padres y unos ingresos familiares inferiores a 20.000 dólares anuales, fueron factores de riesgo significativos en la recurrencia de la deformidad (Avilucea FR, 2009; Panjavi B, 2012; Ramírez $\mathrm{N}, 2011$ ) (20). Se vio que muchas familias tenían dificultades a la hora de comprender el uso de las férulas de abducción así como la importancia y los objetivos del tratamiento, lo que comprometió seriamente el éxito del procedimiento $(1,3)$.

La razón más común del incumplimiento del tratamiento de las férulas es la incomodidad que presentan los niños, esto repercute 
directamente en la adherencia al tratamiento y consecuentemente, en un aumento significativo en el número de recidivas (21).

Las tasas de recurrencia varían entre $8-50 \%$ debido principalmente al incumplimiento y a la falta de técnica a la hora de aplicar el método (Kim S, 2014) (7).

El grupo de la Universidad de Iowa definió recurrencia como la reaparición de cualquiera de los componentes de la deformidad lo que incluye: cavo, equino, antepié adducto y retropié varo (Morcuende JA, 2004) $(20,22)$.

El primer signo de recaída es una disminución de la dorsiflexión en relación a la observada en la anterior valoración. Las recidivas aparecen fundamentalmente en los primeros 5 años de vida coincidiendo con un periodo de rápido crecimiento del pie, rara vez se verá en mayores de 7 años (Ponseti IV, 1996) (23).

Las recidivas se producen a causa de desequilibrio muscular, escasa actividad muscular de los evertores del pie, corrección inadecuada y pérdida de la reducción, lo que da lugar a una deformidad residual que incluye metatarso adducto, contractura del tendón de Aquiles y supinación del antepié en fase de balanceo de la marcha (Opel D, 2014; Gelfer, 2014) $(7,23)$.

En caso de recidiva, primeramente se recomienda continuar con los enyesados semanales, pero ante persistencia de supinación y adducción, o ante deformidades no flexibles, se procederá a realizar la transferencia del tendón tibial anterior a la tercera cuña (Ponseti IV, 1972) (3).

Entre las posibles complicaciones del método Ponseti destacar infección local, irritación cutánea, maceración, abrasión, aparición de ampollas, onicopatías, fibrosis y atrofia muscular de la pierna (Dimeglio et al., 1995) $(3,6,24)$.

El pie equinovaro no tratado produce dolor, deterioro físico y discapacidad, de ahí la importancia de su tratamiento desde temprana edad, preferiblemente después del nacimiento del niño. Además, dado que la tasa de corrección se sitúa entre un $92-100 \%$, se debe priorizar el tratamiento conservador, de manera que se disminuyan las molestias y se favorezca una mejora en la calidad de vida tanto para el niño como para los padres (15).

Debido a que se estima una tasa de éxito del 78\% en el método Ponseti en términos de buenos resultados, recuperación quirúrgica y un número bajo de complicaciones, estos datos motivan a que un amplio número de trabajadores se interesen por esta alternativa. En otras palabras, se trata de un método de bajo coste que sustituye al tratamiento quirúrgico, por lo que la realización de una revisión bibliográfica narrativa acerca de este tratamiento ortopédico despertaba mi interés (4).

\section{Materiales y métodos}

El análisis de la revisión bibliográfica se ha llevado a cabo en dos fases, la primera fase para la localización y selección de los artículos y la segunda para la evaluación de los mismos.

El material bibliográfico se obtuvo a través de la consulta en PubMed. Las palabras claves o descriptores empleados en dichas bases fueron equinovarus, clubfoot y Ponseti method. Estos términos, obtenidos a través el Mesh Database y los Descriptores en Ciencias de la Salud (DeCS), se truncaron mediante el operador booleano "AND" con el fin de obtener los resultados deseados. La estrategia de búsqueda seguida en la mayoría de los buscadores mantenía el siguiente patrón: clubfoot AND Ponseti y equinovarus AND Ponseti method.

La figura 2 clarifica el proceso de la primera fase y los resultados de selección conseguidos. Se obtuvieron doscientos catorce artículos, de éstos ciento ochenta y siete fueron excluidos al no cumplir los criterios de inclusión/exclusión [Ver Tabla 3], finalmente, se incluyeron 27 artículos en la revisión bibliográfica. La evaluación de los estudios escogidos se ha realizado siguiendo la metodología Critical Appraisal Skills Programme español (CASPe), clasificándolos según su evidencia científica. 


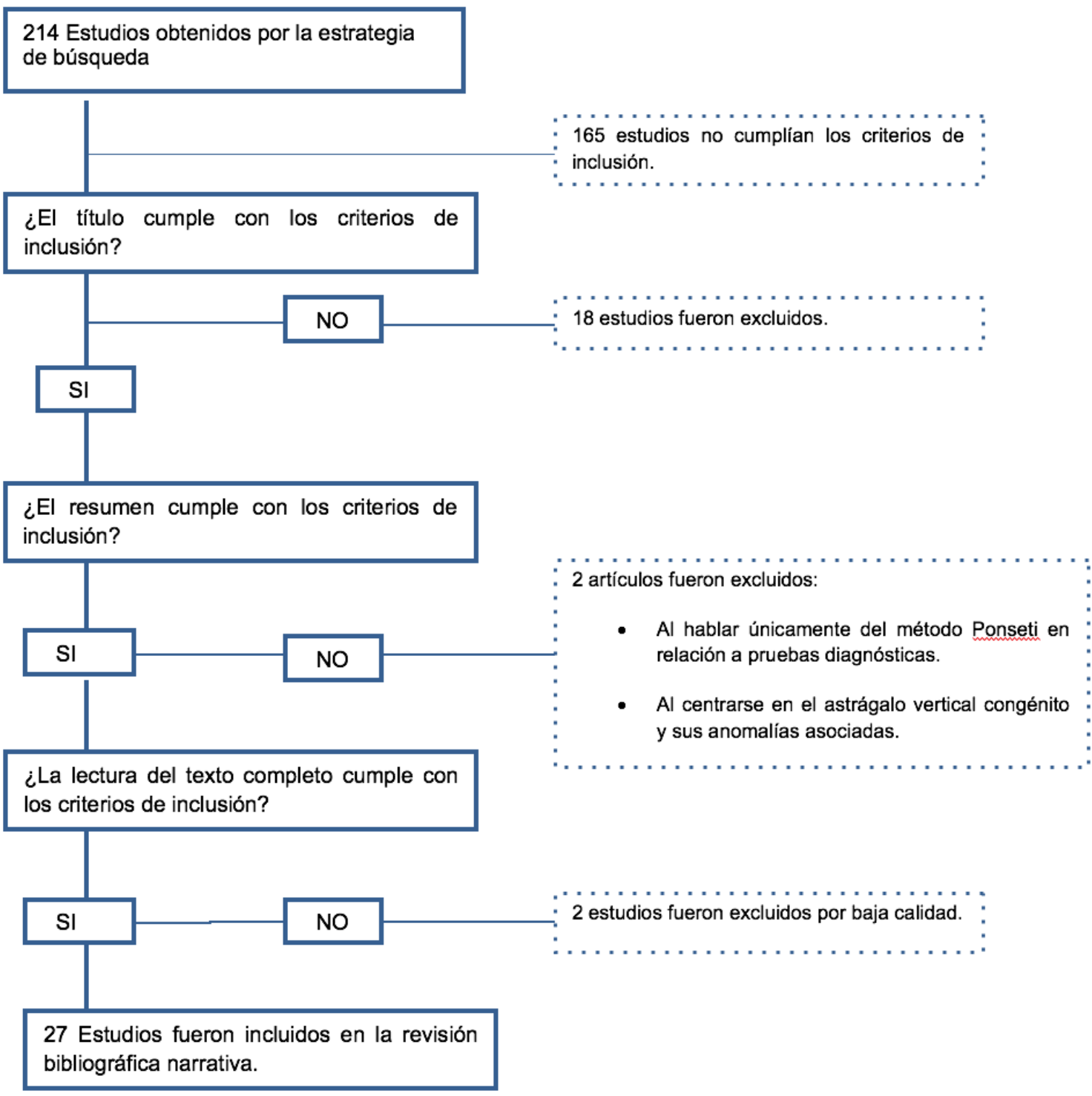

Figura 2. Estrategia de búsqueda

Tabla 3. Criterios de inclusión y exclusión

\begin{tabular}{|c|c|}
\hline Criterios de inclusión & Criterios de exclusión \\
\hline $\begin{array}{l}\text { Tipo de estudio: } \\
\text { - Metaanálisis } \\
\text { - Revisiones sistemáticas } \\
\text { - Ensayos clínicos } \\
\text { Se incluyeron } 4 \text { artículos con una antigüedad } \\
\text { superior a diez años por relevancia. }\end{array}$ & Artículos con antigüedad superior a cinco años. \\
\hline $\begin{array}{l}\text { Participantes: } \\
\text { - Niños con edades comprendidas entre } 1 \text { y } 10 \\
\text { años. } \\
\text { Diagnóstico de pie equinovaro idiopático tanto } \\
\text { unilateral como bilateral. }\end{array}$ & Artículos que carecieran de calidad metodológica. \\
\hline
\end{tabular}




\begin{tabular}{|l|l|}
\hline $\begin{array}{l}\text { Intervención: } \\
\text { - } \quad \text { Enyesado. } \\
\text { - Manipulaciones pasivas varias veces en sema- } \\
\text { nas. }\end{array}$ & $\begin{array}{l}\text { Artículos con un idioma diferente al inglés o } \\
\text { francés. }\end{array}$ \\
\hline $\begin{array}{l}\text { Medida de resultado: } \\
\text { - Apoyo plantígrado del pie } \\
\text { - Reducción de la adducción, equino y varo del } \\
\text { pie. }\end{array}$ & Artículos que carecieran de evidencia científica. \\
\hline
\end{tabular}

\section{Resultados}

A continuación, y de manera desarrollada, se procederá a desarrollar los contenidos de los estudios, primeramente $\mathrm{y}$, de manera individual, los que se han considerado de mayor envergadura; por ello, inicialmente se expondrá un metaanálisis que establece una comparativa de la eficacia entre dos métodos conservadores (método Ponseti y método Kite), en cuanto al tratamiento del pie equinovaro. Seguidamente, se desarrollarán dos revisiones sistemáticas; posteriormente, por medio de gráficos, se describirán los resultados obtenidos tras la búsqueda; es decir, se hará un compendio que responde a los ya mencionados objetivos. Por último, y de forma esquemática, se abordarán los estudios de mayor importancia ordenados según el año de publicación; los resultados de esta revisión se presentan en la tabla 4, la cual muestra el contexto de cada uno de los estudios, así como el diseño, objetivos y las variables de resultado evaluadas.

\section{Metaanálisis}

El objetivo de este metanaálisis, compuesto por 4 artículos, era comparar tanto el método Ponseti como el método Kite, para evidenciar cuál de ellos era el más efectivo en el tratamiento del pie equinovaro. Se incluyeron todos aquellos estudios compuestos por niños menores de 6 meses que presentaran la deformidad de pie equinovaro idiopático.

Estos 4 artículos muestran una tasa combinada global de corrección de la deformidad del $92,37 \%$ en el grupo tratado con el método Ponseti frente a un $39,68 \%$ en el grupo tratado con el método Kite. Teniendo en cuenta los pies no corregidos y la tasa de recaídas conjuntamente como casos sin éxito, el grupo Ponseti alcanzó tasas de éxito de $76,65 \%$, mientras el grupo Kite alcanzó sólo el $38,73 \%$.
- Ippolito y cols. Consideraron un fallo en el tratamiento en grupo tratado con el método Marino-Zucco (método muy parecido al método Kite) a aquellos pies que mostraron resistencia a la corrección después de 12 semanas. En este grupo de 47 pies, el fallo ocurrió en 16 y 22, de 47 recayeron a una edad promedio de 3 años. En el grupo tratado mediante el método Ponseti se consideró fracaso cuando los pies mostraban resistencia a la corrección después de 6-8 semanas y recaída cuando la deformidad reaparecía después de los 4 años de edad. En este grupo el fallo ocurrió en 5 pies y 20 de 49 pies recayeron. La tasa de recaídas fue similar en ambas modalidades, sin embargo el método Ponseti experimentaba mayores rangos de movimiento de tobillo, especialmente a la DF.

- Sud y cols, consideraron un fallo en el tratamiento cuando el pie no alcanzó la corrección después de 1 año. En el grupo tratado con el método ponseti se corrigieron 33 pies de 36; mientras que en el grupo tratado mediante el método Kite se corrigieron 21 de 31 pies. Hubo 7 recaídas tanto en el grupo Ponseti como en el grupo Kite. Los autores también concluyen que las deformidades residuales en el grupo tratado con el método Kite muestran preponderancia en el retropié. También se sugiere que los mejores resultados en la PG pueden estar asociados a la cirugía mínimamente invasiva del tendón de Aquiles. No hay diferencias estadísticamente significativas entre ambos tratamientos en casos leves o moderados; sin embargo, los casos muy severos tuvieron mejores resultados cuando se trataron con el método Ponseti.

- Herzenberg y cols. consideraron un fallo en el tratamiento conservador cuando la resis- 
tencia a la corrección ocurría después de 12 semanas. Hubo 33 de 34 correcciones en cuanto al método Ponseti y 2 de 34 en el método Kite. Hubo 33 de 34 correcciones en cuanto al método Ponseti y 2 de 34 en el método Kite.

- Segev y cols, se consideró fallo en el tratamiento cuando el autor principal decidió realizar una cirugía en función del grado de corrección logrado tras las manipulaciones. 35 de 61 se sometieron a tratamiento quirúrgico tras ser tratados con el método Kite y 45 de 48 en el caso de los tratados mediante el método Ponseti. 35 de 61 se sometieron a tratamiento quirúrgico tras ser tratados con el método Kite y 45 de 48 en el caso de los tratados mediante el método Ponseti.

Se concluyó que había una diferencia significativa entre los dos grupos, resultando el tratamiento mediante el método Ponseti el más efectivo. Asímismo detectaron limitaciones metodológicas como por ejemplo, un tamaño de muestra reducido (25).

\section{Revisiones sistemáticas}

- Revisión sistemática realizada en Sudáfrica, 2017: engloba un total de 27 estudios, de los cuales sólo 22 cumplían los criterios de inclusión con una franja tem- poral de 17 años. En relación a los sujetos, se incluyeron desde recién nacidos hasta niños de 10 años. Los objetivos principales de esta revisión fueron determinar y evaluar el éxito del método Ponseti en Sudáfrica. Esta revisión sugiere que el método Ponseti parece dar una corrección exitosa del pie equinovaro cuando se mide a través de evaluación clínica simple, por la puntuación de Pirani (fue la más utilizada) o por la clasificación de Dimeglio. En conclusión, los estudios incluidos reportan éxito en el $68-98 \%$ de los casos después de la fase de corrección (15).

- Revisión sistemática realizada en la India, 2018: engloba un total de 10 estudios en los que incluye casos de niños mayores de 2 años cuyo pie equinovaro no fue tratado, estableciendo como objetivo principal el de determinar la eficacia del tratamiento del pie equinovaro en niños "mayores" que es lo que el autor define como "pie equinovaro desatendido". En cuanto a las limitaciones encontradas, el autor resalta la necesidad de la unificación de criterios para poder extraer conclusiones contundentes. Pese a esto, concluye que el tratamiento del pie zambo es efectivo en mayores de 2 años, si bien, el límite de edad de inicio del tratamiento para que éste sea exitoso sigue siendo incierto (19).

\section{Agrupación de datos tras la búsqueda}

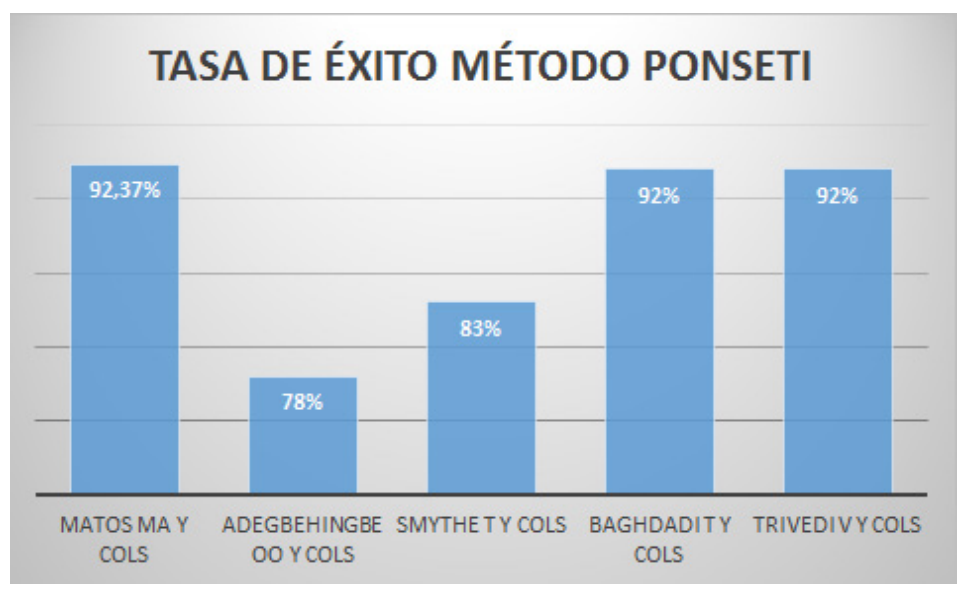

Tasa de éxito del Método Ponseti según autor 


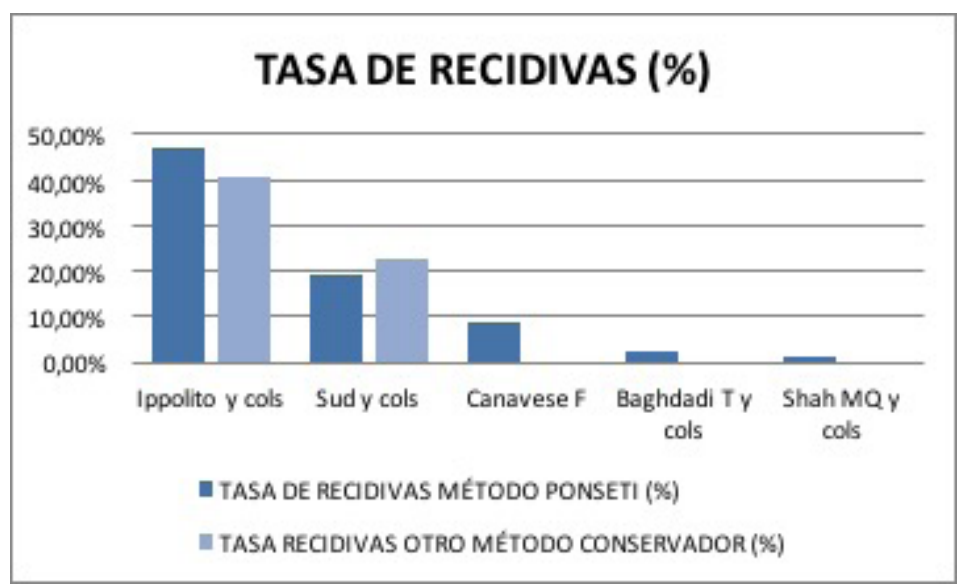

Tasa de recidivas del método Ponseti y método Kite según autor

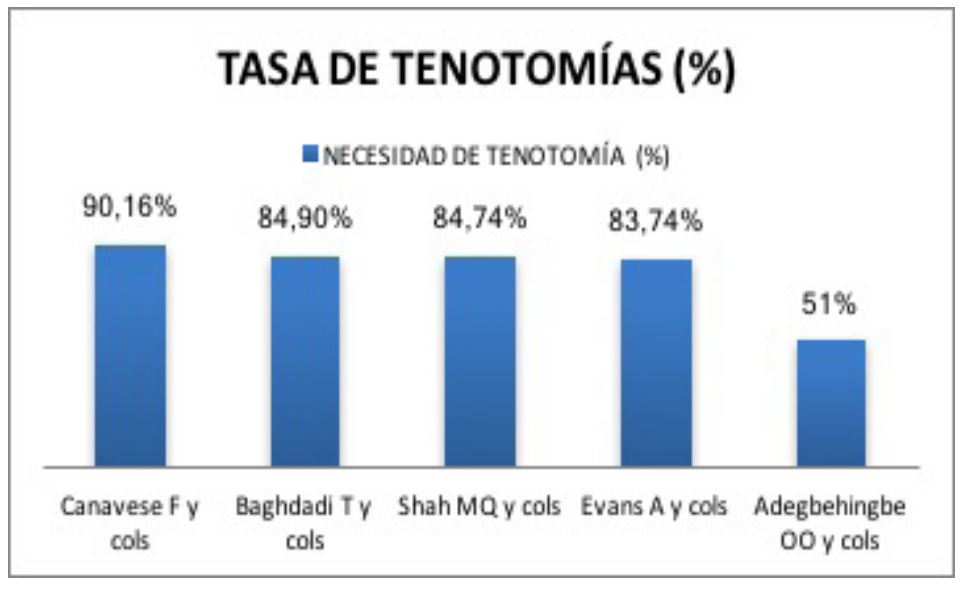

Necesidad de tenotomías tras método Ponseti según autor

\section{TASA DE INCUMPLIMIENTO (\%)}

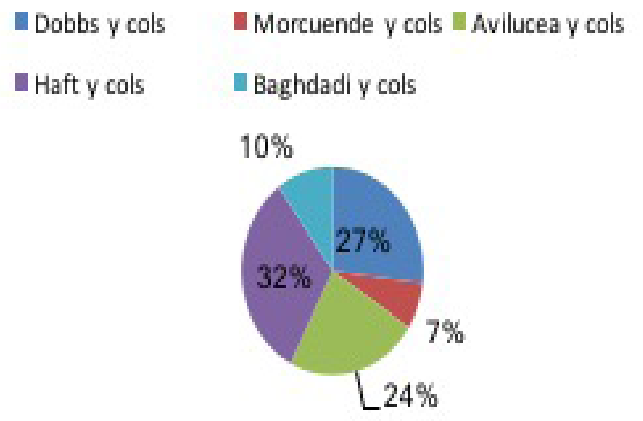

Falta de adherencia al tratamiento conservador según autor (21) 


\section{Discusión}

A pesar de la existencia de numerosas terapias conservadoras, hay cierta discrepancia respecto a cuál es la más adecuada. Richards, Steinman, Faulk y colaboradores, compararon el método francés y el Ponseti para el tratamiento del pie equinovaro idiopático y no encontraron diferencias significativas entre ambos procedimientos; ambas técnicas proporcionan una excelente corrección inicial, una tasa de recaídas similar y resultados comparables. Según Wicart y Chotel, aunque los resultados entre ambas técnicas fueran similares, sí encontraron mayores tasas de cirugía en el método francés en comparación con el método Ponseti. Sin embargo, estos estudios demuestran que ninguno de los dos métodos elimina la necesidad de una liberación posteromedial. El método híbrido combina las ventajas de ambos sistemas, fusionándolos; éste fue capaz de corregir $12 / 20$ pies equinovaros y se reportó un menor porcentaje de cirugías y de liberación posteromedial (Faulks S, 2009; Bensahel H, 1994; Richards S, 2008; Steinman S, 2009) (6). Tras la revisión de la literatura, Balasankar $\mathrm{G}$ y colaboradores, analizaron y compararon varios métodos conservadores para el tratamiento del pie zambo y concluyeron que hoy en día, el método Ponseti se ha convertido en la mejor opción en términos de buenos resultados, bajo coste y bajo uso de tecnología (8).

Ciertos estudios, han subrayado la eficacia del tratamiento incluso en pacientes con una presentación tardía de la deformidad o con una deformidad severa (Dobbs MB, 2009; Laurenco AF, 2007; Evans AM, 2009) (12); contrariamente, Kims S y Opel D en 2014, expusieron, que el tratamiento en niños mayores de 4 años era sinónimo de fracaso (7). Radler $\mathrm{C}$ coincide en que una deformidad compleja del pie zambo supone un desafío para el profesional que lo va a manejar, pero insiste en que es posible con un buen aprendizaje y entrenamiento (3). Según los resultados del estudio realizado por J. Jochymek et al., los pacientes con una deformidad severa de pie equinovaro requirieron un mayor número de yesos correctores así como el $80 \%$ precisaron de una tenotomía percutánea del Tendón de Aquiles (17).

\section{Conclusion}

El método Ponseti se considera el gold standard en el tratamiento del pie equinovaro debido a su eficacia y seguridad; sin embargo, todavía existe mucha controversia en cuanto a la etiología, anatomía patológica, clasificación, tratamiento y criterios de evaluación.

Debido a la heterogeneidad de los estudios encontrados en cuanto a metodologías utilizadas, fechas y áreas de trabajo, la comparación de los estudios ha resultado compleja. La gran mayoría de los estudios expone claramente los objetivos, así como las características personales de los pacientes y la intervención a la que fueron sometidos. No obstante, encontramos limitaciones tales como la falta de estudios que hablaran de las dificultades encontradas por los padres a la hora de aplicar las férulas de abducción o los efectos adversos derivados del método Ponseti. Otra limitación encontrada fue la ausencia de la definición de fracaso de tratamiento y sus correspondientes motivos. Así todo, con los datos actuales, se puede afirmar que un insuficiente tiempo de uso de las ortesis de mantenimiento, su uso inadecuado, así como el incumplimiento del tratamiento, dan como resultado un aumento en el riesgo de recaída de la deformidad. Sería atrayente la realización de estudios que midiesen el grado de deformidad con la misma escala de clasificación y estudios que definieran qué método presenta mayores ventajas.

El papel del podólogo en relación al pie zambo es fundamental a la hora de detectar de manera precoz la deformidad y minimizar posibles alteraciones del patrón de la marcha así como alteraciones psicológicas del sujeto. Al tratarse de una afección común que afecta a 1 de cada 1000 recién nacidos, es de suma importancia una completa formación teórica y práctica para así convertirnos en verdaderos especialistas. El dominio del método Ponseti, supone un gran reto para el podólogo puesto que esto garantizaría la excelencia del tratamiento. Nuestro papel es primordial para cooperar con los padres y proporcionarles información a la hora de la prevención de factores predisponentes durante el embarazo, así como durante la colocación del calzado/ botas correctoras tipo Bebax o Denis Browne. Otro de los cometidos sería minimizar las 
prácticas intervencionistas e invasivas, tales como la cirugía, así como fomentar la creación de un equipo multidisciplinar junto con médico rehabilitador, pediatras, fisioterapeutas y ortopedas.

En definitiva, se puede decir que el método Ponseti es el método de referencia en el tratamiento de la deformidad de pie equinovaro, cuyo éxito está directamente relacionado con la adherencia al tratamiento por parte del paciente y sus padres. En relación a la edad de inicio óptima para que el tratamiento conservador resulte efectivo, se ha visto que lo aconsejable sería tras el nacimiento; y aunque todavía no hay un establecimiento claro del límite de edad, hay casos de niños mayores de
2 años donde el tratamiento produjo resultados satisfactorios a largo plazo. Constatar que la opción quirúrgica, no deja de entrañar un riesgo para el paciente ya que va de la mano de una cicatriz más o menos extensa, rigidez, debilidad y dolor. Esta terapia, quedaría reservada para aquellos niños que tras agotar las opciones conservadoras, presenten una deformidad residual severa. Hasta la fecha, no existe suficiente evidencia científica de que la fusión de varios métodos conservadores pueda presentar más beneficios que una única técnica aislada. En conclusión, el método Ponseti supone una interesante alternativa que cobra aún poca importancia debido en parte al desconocimiento de profesionales y usuarios.

\section{Bibliografía}

1. Jaqueto PA, Martins GS, Mennucci FS, Bittar CK, Zabeu JLA. Functional and clinical results achieved in congenital clubfoot patients treated by Ponseti's technique. Rev Bras Ortop. 2016;51(6):657-61.

2. Shabtai L. Worldwide spread of the Ponseti method for clubfoot. World J Orthop. 2014;5(5):585.

3. Radler C. The Ponseti method for the treatment of congenital club foot: review of the current literature and treatment recommendations. Int Orthop [Internet]. 2013;37(9):1747-53.

4. Vigouroux F, Bertani A, Cunin V, Mathieu L, Launay F, Rongieras F. Traitement du pied bot varus équin idiopathique Mise en place de la méthode Ponseti dans un pays émergent. Med Sante Trop [Internet]. 2016;26(1):24-30.

5. Baghdadi T, Bagheri N, Najafi A, Mansouri P, Farzan M. Ponseti Casting Method in Idiopathic Congenital Clubfoot and Its Correlation with Radiographic Features Abstract. Arch bone Jt Surg [Internet]. 2017;5(3):168-73.

6. Canavese F, Mansour M, Moreau-Pernet G, Gorce Y, Dimeglio A. The hybrid method for the treatment of congenital talipes equinovarus. J Pediatr Orthop B [Internet]. 2017;26(3):197-203.

7. Shah MQ, Khan A, Zardad MS, Iqbal R, Ahmed S. Ponseti Technique For Management Of Congenital Idiopathic Club Foot. J Ayub Med Coll Abbottabad [Internet]. 2017;29(2):246-9.

8. Balasankar G, Luximon A, Al-Jumaily A. Current conservative management and classification of club foot: A review. J Pediatr Rehabil Med [Internet]. 2016;9(4):257-64.

9. Gurnett CA, Boehm S, Connolly A, Reimschisel T, Dobbs MB. Impact of congenital talipes equinovarus etiology on treatment outcomes. Dev Med Child Neurol. 2008;50(7):498-502.

10. Palma M, Cook T, Segura J, Pecho A, Morcuende JA. Descriptive epidemiology of clubfoot in Peru: a clinic-based study. Iowa Orthop J [Internet]. 2013;33:167-71.

11. Stone P, Martis W, Crawford H. Idiopathic congenital talipes equinovarus; not always an isolated anomaly. A review of long-term outcomes. J Matern Neonatal Med [Internet]. 2017;0(0):1-6.

12. Grimes CE, Holmer H, Maraka J, Ayana B, Hansen L, Lavy CBD. Cost-effectiveness of club-foot treatment in low-income and middle-income countries by the Ponseti method. BMJ Glob Heal [Internet]. 2016;1(1):e000023.

13. Liu ZY, Li XD, Chen B, Zheng CY, Zhong YS, Jia YL, et al. Retinoic acid retards fetal and hindlimb skeletal development asymmetrically in a retinoic acid-induced clubfoot model. Exp Toxicol Pathol [Internet]. 2010;62(6):663-70.

14. Cosma D, Vasilescu DE. A Clinical Evaluation of the Pirani and Dimeglio Idiopathic Clubfoot Classifications. J Foot Ankle Surg [Internet]. 2015;54(4):582-5.

15. Smythe T, Mudariki D, Kuper H, Lavy C, Foster A. Assessment of success of the Ponseti method of clubfoot management in sub-Saharan Africa: a systematic review. BMC Musculoskelet Disord [Internet]. 2017;18(1):453.

16. Wainwright AM, Auld T, Benson MK, Theologis TN. The classification of congenital talipes equinovarus. J Bone Joint Surg Br [Internet]. 2002;84(7):1020-4.

17. Jochymek J, Turek J. The ultrasonography evaluation of talar dysplasia as a potential prognostic factor for predicting the course and outcomes of clubfoot deformity treatment using Ponseti technique. Acta Orthop Traumatol Turc [Internet]. 2018;1-5. 
18. Mejabi JO, Esan O, Adegbehingbe OO, Asuquo JE, Akinyoola AL. A prospective cohort study on comparison of early outcome of classical Ponseti and modified Ponseti post tenotomy in clubfoot management. Ann Med Surg [Internet]. 2017;24:34-7.

19. Digge V, Desai J, Das S. Expanded Age Indication for Ponseti Method for Correction of Congenital Idiopathic Talipes Equinovarus: A Systematic Review. J Foot Ankle Surg [Internet]. 2018;57(1):155-8.

20. Zhao D, Liu J, Zhao L, Wu Z. Relapse of Clubfoot after Treatment with the Ponseti Method and the Function of the Foot Abduction Orthosis. Clin Orthop Surg [Internet]. 2014;6(3):245.

21. Zhao D, Li H, Zhao L, Liu J, Wu Z, Jin F. Results of clubfoot management using the Ponseti method: Do the details matter? A systematic review [Internet]. Vol. 472, Clinical Orthopaedics and Related Research. 2014;1329-36.

22. Liu Y-B, Jiang S-Y, Zhao L, Yu Y, Zhao D-H. Can Repeated Ponseti Management for Relapsed Clubfeet Produce the Outcome Comparable With the Case Without Relapse? A Clinical Study in Term of Gait Analysis. J Pediatr Orthop [Internet]. 2017;0(0):1.

23. Hosseinzadeh P, Kiebzak GM, Dolan L, Zionts LE, Morcuende J. Management of Clubfoot Relapses With the Ponseti Method: Results of a Survey of the POSNA Members. J Pediatr Orthop [Internet]. 2017;25(3):195-203.

24. Andreoli E, Troiani A, Tucci V, Barlafante G, Cerritelli F, Pizzolorusso G, et al. Osteopathic manipulative treatment of congenital talipes equinovarus: A case report. J Bodyw Mov Ther [Internet]. 2014;18(1):410.

25. Matos MA, de Oliveira LAA. Comparison between Ponseti's and Kite's clubfoot treatment methods: a meta-analysis. J Foot Ankle Surg [Internet]. 2010;49(4):395-7.

26. Trivedi V, Badhwar S, Dube AS. Comparative Analysis between Podography and Radiography in the Management of Idiopathic Clubfeet by Ponseti Technique. J Clin Diagn Res [Internet]. 2017;11(2):RC09$\mathrm{RC} 12$.

27. Evans A, Chowdhury M, Rana S, Rahman S, Mahboob AH. "Fast cast" and "needle Tenotomy" protocols with the Ponseti method to improve clubfoot management in Bangladesh. J Foot Ankle Res [Internet]. 2017;10(1):49. 